2. They are more vicious when handled and bite much more freely than normal animals.

3. Ferrets develop real hysteria in the sense of charging round the cages and running into the cage sides.

4. They develep true epileptiform fits when severely affected.

On the whole, however, ferrets do not appear to be quite so susceptible to these nervous abnormalities as do dogs. On the other hand, they have the advantage that experimental work can be done with much smaller quantities of the toxic agent.

Messrs. Wallace and Tiernan kindly lent the apparatus for agenizing flour on a laboratory scale. The flour used in most of the experiments reported here was treated in the laboratory, whereas that used in those described in the 1946 paper was done in the mill.

REFERENCES

Kent-Jones, D. W., and Herd, C. W. (1930). J. Soc. chem. Ind. 49, 223.

Mellanby, E. (1946). British Medical Journal, 2, 885

Melnick, D. and Cowgill, G. R. (1937) J. Nutrit, 14, 401.

Wagner, J. R., and Elvehjem, C. A. (1944). Ibid., 28, 431.

\section{PERFORATED PEPTIC ULCER}

\section{SHORT-TERM VARIATIONS IN FREQUENCY}

BY

\section{R. A. JAMIESON, F.R.C.S.}

Lecturer in Surgery, University of Glasgow

(From the Peptic Ulcer Clinic, Western Infirmary, Glasgow)

In a previous paper (Illingworth, et al., Journal, 1944, 2, $617,655)$ attention was drawn to certain seasonal, weekly, and daily variations in the frequency of perforated peptic ulcer.

The purpose of the present paper is to examine these short-term variations more fully, to enlarge the data previously given, and to add fresh observations on the influence of meal hours and of physical stress in determining the moment of perforation. The material comprises all cases of perforation treated in the three main Glasgow Hospitals (Royal, Western, and Victoria Infirmaries) during the period 1924-45: The patients are drawn from a heavily populated but geographically small area of the West of Scotland, and the majority are engaged in heavy industry.

In 1938 and again in 1943 we established that the three main hospitals treated $80 \%$ of all cases dealt with in the district, and our knowledge of local conditions convinced us that this proportion was a reasonable estimate for the whole period covered by the survey. The exclusion of the remaining $20 \%$ of perforations is not likely to be a serious cause of error for the present purpose, because there is no policy of preferential admission to the smaller institutions in certain months, on certain days, or at certain hours.

\section{Results}

Seasonal Variation.-Perforations occurring in like months were added together and the result is given in Fig. 1. It is seen that the incidence of perforation remains re m a r kably uniform during the spring and summer, but falls off strikingly in a $u \mathrm{t} \mathrm{u} \mathrm{m} \mathrm{n}$ ( A u gus t, Sep te m ber, O c t o b e r), a n d finally reaches a high peak in Decem ber. The foci of

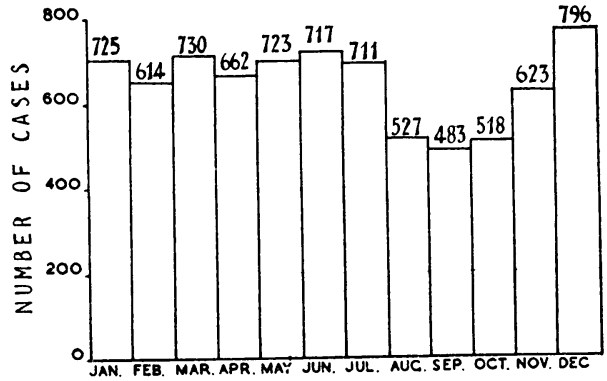

Fig. 1.-Perforated peptic ulcer; incidence by months (1924-45). The chart is adjusted to standard 30-day months; the numerals give the actual cases observed.

chief interest are the transition periods July-August and October-November, together with the December peak.

In order to study these periods more closely we regrouped our cases by calendar weeks (instead of by calendar months), and the result is given in Fig. 2. It will be seen that the incidence of perforation is somewhat high in the first two weeks of July and then begins to fall off to the low level of autumn: the fact that the fall begins in the second half of July is of some interest. July is the popular holiday month in the West of Scotland, and the great majority of the artisan and labouring class are on holiday during the "Glasgow Fair," which lasts for ten days during the second and third or the third and fourth weeks of July. It seems possible that the period of rest may be responsible for a degree of immunity from perforation in the ensuing months. The lowest weekly incidence of perforation has been reached by mid-September, and thereafter the incidence gradually rises till the spring and summer level has been regained by mid-December. Again, the December peak is seen to be due not to a uniformly high incidence throughout the month but to a high incidence in the later weeks : the appearance of a sudden transition to a high incidence at the middle of the month is, however, an artefact attributable to arbitrary grouping in seven-day periods.

The nature of the December peak is better seen in Fig. 3, which shows the number of perforations by individual days from Dec. 1 to Jan. 15 (perforations occurring on like days throughout the 22-year period being added together). It will be seen that there are wide fluctuations from day to day, but the trend as a whole is quite clear-namely, a steady increase throughout the month.

It is worth noting that there is no special peculiarity about the number of perforations in the Christmas-New Year week, and this fact weighs against dietary or alcoholic excesses being responsible for the high incidence of per-

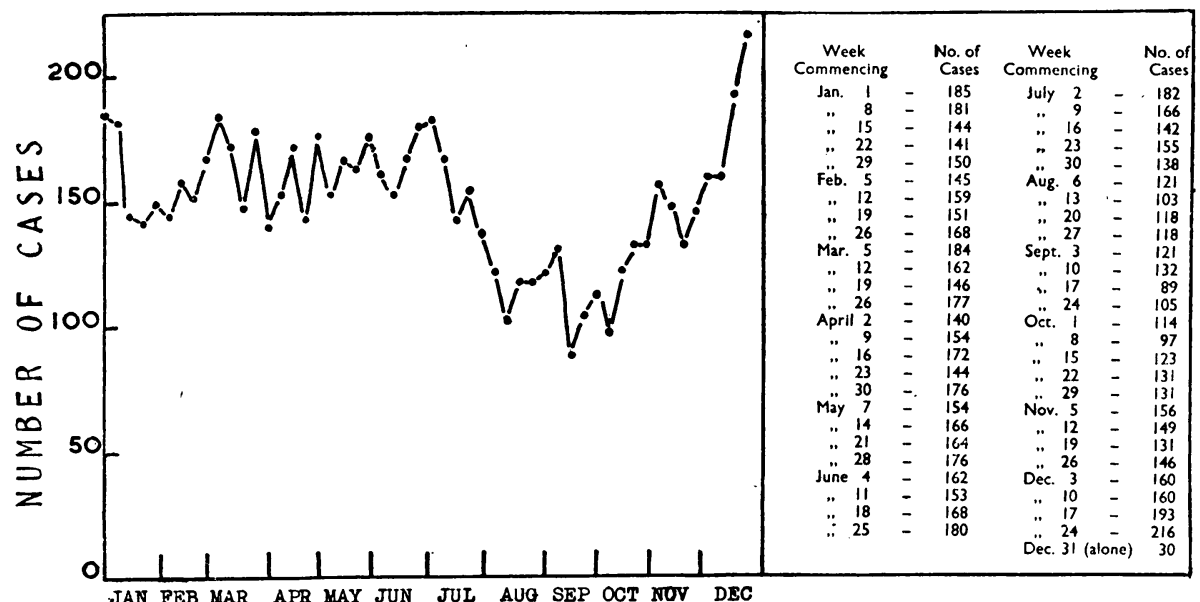

Fig. 2.-Perforated peptic ulcer; incidence by calendar weeks (1924-45) 


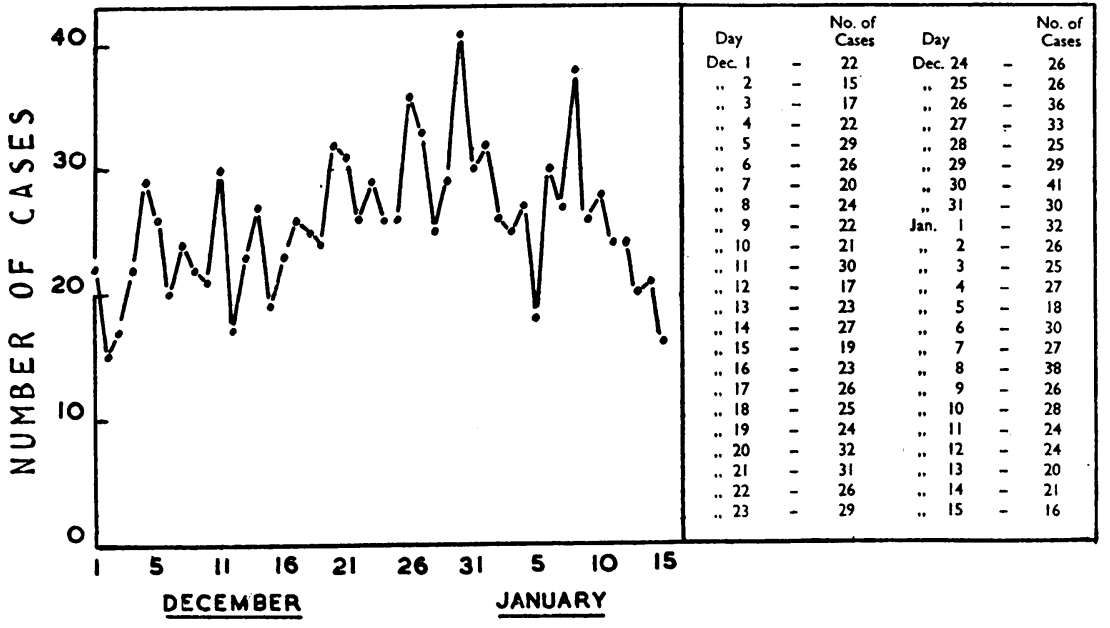

FIG. 3.-Perforated peptic ulcer; incidence by single days (1924-45). delay in admission at the week-end than during the week was excluded by examining a sample of patients (Western Infirmary, 1938-45): the intervals between perforation and operation were in close agreement.

Daily Variation.-Perforation does not take place with uniform frequency throughout the day, as we were able to show previously with a sample of some 900 patients. We have extended this observation to a larger series comprising all patients treated at the Western Infirmary during the period 1938-45. It is relevant at this point to state that perforation of a peptic ulcer occurs with such dramatic suddenness and is accompanied by such intense pain that there is generally little difficulty in determining the precise time of onset. Of 1,348 perforations the time of perforation was recorded in 1,169 (Fig. 5). It will be seen that perforation is rare during the night, gradually increases in frequency during the morning, reaches a peak in the middle of the afternoon, foration. Again, it is perhaps relevant to point out that in Scotland there is more merrymaking at the New Year than at Christmas, and that in the first days of January there is actually a fall in the incidence of perforation from the high level reached by the end of December. We conclude, therefore, that the December peak cannot be attributed to seasonal festivity, but we are unable to suggest an alternative explanation.

Weekly Variation.-In our previous report we showed that perforation did not occur with uniform frequency throughout the week, but that there was a deficit of perforations on Sundays and Mondays. It seemed useful to extend this observation (which was based on a sample of patients treated during the war years). We have therefore classified all our patients by the day of the week they were admitted to hospital, and perforations admitted on like days throughout the 22-year period have been added together. The result is given in Fig. 4. The suggestion that there is a deficit of perforations on Sundays and Mondays is fully borne out by this large series, and moreover it is seen that there is a fairly steady increase in perforations towards the end of the week. This finding seems to be compatible with the suggestion previously offered, that rest of the week-end might be responsible for a measure of immunity from perforation in the ensuing days.

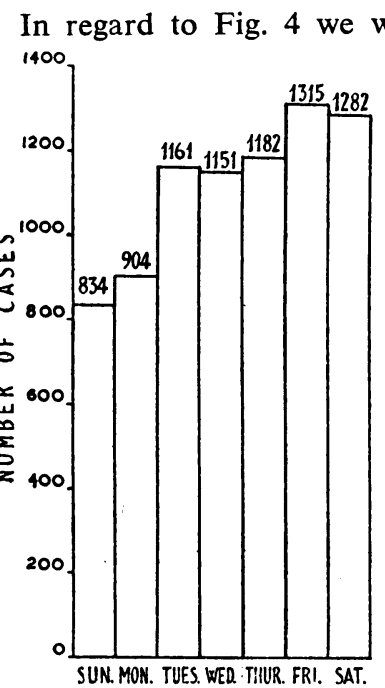

Fig. 4.--Perforated peptic ulcer; incidence by days of week.

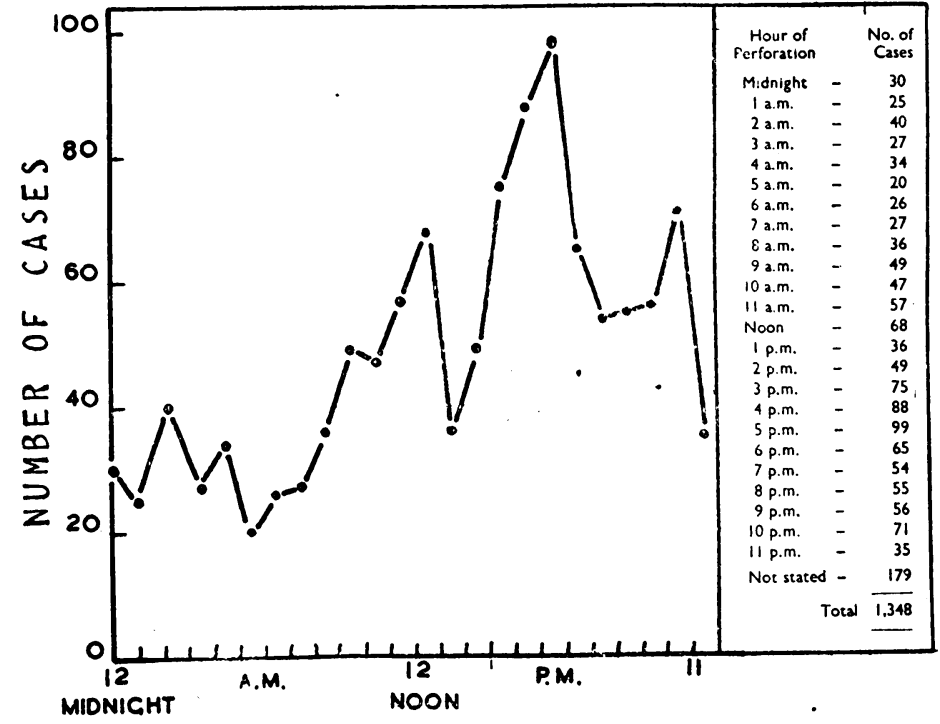

FIG. 5.-Perforated peptic ulcer; hour of perforation.

and again diminishes in frequency during the evening. It is noteworthy that the frequency is greatest towards the end of the working day and is lowest during the period of rest. This may be compared with the low incidence after the summer holiday period and the week-end rest.

In addition to the influence of rest on the daily variation in frequency two other factors merit inquiry: these are the influence of meal-hours and of physical stress. These factors were inquired into during the interview of 537 patients who had previously suffered perforation (Western Infirmary patients treated during the period 1938-45).

The relation of the moment of perforation to the time of the last meal is given in Fig. 6 . It will be seen that perforation was most frequent between two and three hours

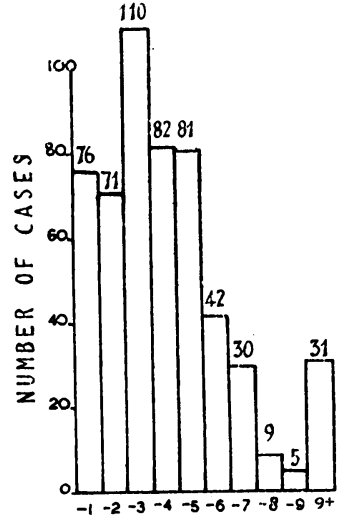

FIG. 6.-Interval (in hours) between perforation and last meal. 
after a meal. Some such relationship is, however, a natural consequence of the fact that the peak incidence of perforation is in the middle of the afternoon and that the majority of patients have dinner at noon. A better idea of the relationship between perforation and the last meal is obtained by considering separately those patients who perforated $(a)$ between 6 a.m. and noon, $(b)$ between noon and 6 p.m., and $(c)$ between 6 p.m. and midnight. These groups are represented in Fig. 7, where it will be seen that there

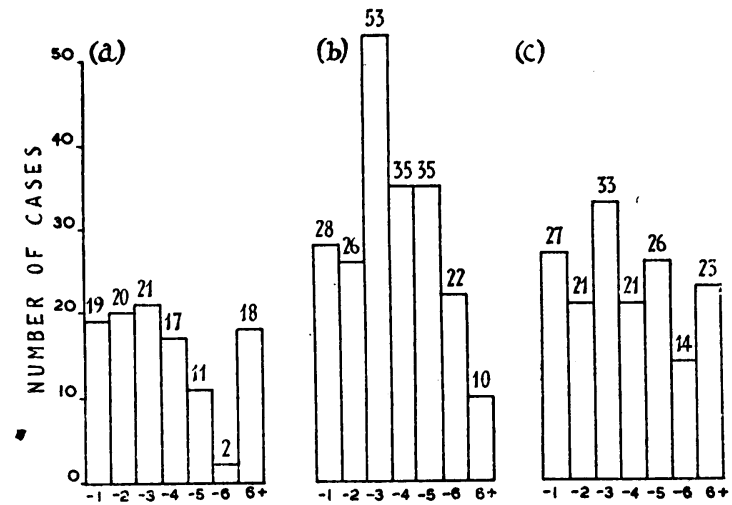

FIg. 7.-Number of hours between perforation and last meal in patients perforating between $(a) 6$ a.m. and noon;

(b) noon and 6 p.m. ; $(c) 6$ p.m. and midnight.

is no correspondence among them as regards a peak incidence related to the preceding meal. We concluded that there was no evidence to suggest that perforation occurs at any particular phase of digestion.

Finally we investigated the possibility that perforation might be precipitated by strenuous bodily exertion. We were able to ascertain what the patients were doing at the precise moment of perforation in 504 cases, thus :

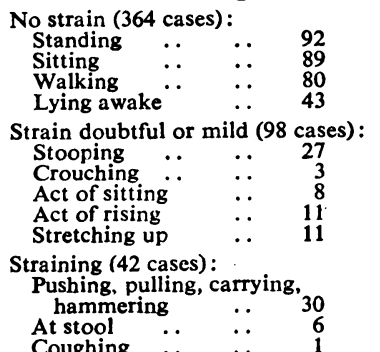

\begin{tabular}{|c|c|}
\hline $\begin{array}{l}\text { Sleeping } \\
\text { Washing, shaving } \\
\text { Kneeling } \quad .\end{array}$ & $\ddot{0}$ \\
\hline $\begin{array}{l}\text { Eating } \\
\text { Drinking } \ldots \\
\text { Turning in bed } \\
\text { Having barium mea }\end{array}$ & $\begin{array}{l}\ddot{a} \\
\dot{a}\end{array}$ \\
\hline $\begin{array}{l}\text { Playing football } \\
\text { Jumping } \\
\text { Leaning on stomad }\end{array}$ & to \\
\hline
\end{tabular}

From the foregoing it is clear that severe exertion plays little part in precipitating perforation. Indeed, there is a strong impression, especially on reading the detailed records, that the patients were engaged in all the normal activities of daily life when the pain of perforation struck them.

\section{Summary}

Attention is drawn to the short-term variations in frequency of perforated peptic ulcer as seen in a large series of cases treated in Glasgow hospitals in the period 1924-45.

1. Perforations are relatively uncommon in August, September, and October, and unduly common in December. The beginning of the autumn fall corresponds with the local holiday period at the end of July, and may be due to the period of rest. The December peak is not due to over-indulgence al the Christmas period.

2. Perforations are relatively uncommon on Sundays and Mondays, and become more common towards the end of the week. This may be related to rest at the week-end.

3. Perforations are uncommon during the night. The frequency of perforation increases towards the end of the morning, drops in the early afternoon, and again increases notably towards the end of the afternoon. These changes may well be related to periods of rest and fatigue.

4. No evidence was found that perforation was related to the phase of digestion or occurred during strenuous bodily exertion.

\section{SOME CLINICAL OBSERVATIONS ON THE PRESENT OUTBREAK OF ACUTE POLIOMYELITIS}

\author{
BY
}

\author{
W. HOWLETT KELLEHER, M.D., D.P.H.* \\ Medical Superintendent, Western Hospital, London
}

When many cases of poliomyelitis are occurring, as in the present epidemic, a varied clinical picture is presented. Confusion may arise because, with emphasis on damage to the cranial nerves, other forms of meningo-encephalitis of unknown aetiology may be simulated. The percentage of bulbar cases varies in different epidemics. In the hospital of which I am medical superintendent we have been struck by the comparatively high percentage of cases showing bulbar involvement as compared with our previous experience of the disease. Had the symptoms remained entirely brain-stem, with drowsiness a marked feature and an absence of pareses or palsies of the usual asymmetrical distribution of poliomyelitis, there would have been justification for believing that intermixed with unmistakable cases of poliomyelitis were examples of a virus meningoencephalitis, possibly in no way related in its causal agent to poliomyelitis. From a close study of some of our cases, however, there appears to be little doubt that the cases with which we are dealing, with marked meningeal and bulbar inflammation, are in fact cases of true poliomyelitis. In only a small number was there drowsiness at onset, or even during the course of the illness. Clouding of consciousness and confusion, which appeared in a few cases, amounted to no more than the delirium seen in many acute toxic illnesses. With close observation of the progress of the cases and the ultimate finding of paresis and paralysis there was little doubt that the cause of the illness was the poliomyelitis virus.

\section{Degrees of Attack and Age Incidence}

All degrees of attack are experienced, from the case in which there is only a mild transient illness, simulating other minor illnesses such as the common cold, to the most extreme forms of paralysis involving vital centres in the medulla and causing death within a few hours. The encephalitic or bulbar types may be missed in their minor form but for the occurrence of a frank case of poliomyelitis in another member of the family.

The following cases might be quoted as exemplifying variation in degree of attack :

Two cases were admitted from one family-a woman aged 31 , and her child aged 4. Had the child's been an isolated case, with no known contacts, much difficulty might have been caused in diagnosis. The child, 12 days before admission, complained of headache and pain in right leg and abdomen and had vomited. On admission temperature was normal and the child was apparently well and active, but there was distinct weakness of the right facial muscles. There was no nuchal rigidity or stiffness of spine, and Kernig's sign was absent. More complete examination, however, showed weakness of the right masseter and right sternomastoid. Cerebrospinal fluid and colloidal gold reaction-no abnormality. Diagnosis was made easier by the admission on the same day of the mother with definite poliomyelitis. She had almost complete flaccid paralysis of both lower limbs and lower part of rectus abdominis. Her symptoms had begun with abdominal pain and backache three days before admission; 24 hours before admission she had

* Editor's Note.-Dr. Kelleher, who has had special experience of poliomyelitis during the present epidemic, has, at a time of great pressure, kindly consented to write this article at very short notice in order to afford some guidance to medical men with less experience. For any incompleteness of presentation editorial exigency must be pleaded.-ED., B.M.J. 